\title{
Tapinarof Cream 1\% for Extensive Plaque Psoriasis: A Maximal Use Trial on Safety, Tolerability, and Pharmacokinetics
}

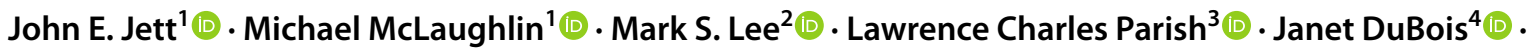

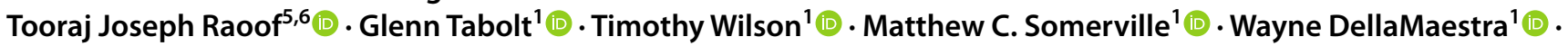 \\ Stephen C. Piscitelli ${ }^{1}$ (i)
}

Accepted: 26 September 2021 / Published online: 28 October 2021

(c) The Author(s) 2021

\begin{abstract} treatment of psoriasis and atopic dermatitis. in patients with extensive plaque psoriasis.

ClinicalTrials.gov Identifier NCT04042103.

\section{Introduction}

Psoriasis is a chronic relapsing inflammatory skin disease, with a prevalence of approximately $2 \%$ in Europe and North America $[1,2]$. Psoriasis is present equally in both sexes, although men have a higher incidence of more severe forms.
\end{abstract}

Background Tapinarof is a novel topical therapeutic aryl hydrocarbon receptor modulating agent in development for the

Objective This multicenter, open-label trial assessed the safety, tolerability, pharmacokinetics (PK), and efficacy of tapinarof cream $1 \%$ once daily (QD) under maximal use conditions in extensive plaque psoriasis.

Methods Adults with a baseline Physician Global Assessment (PGA) score of $\geq 3$ and body surface area (BSA) involvement $\geq 20 \%$ received tapinarof cream $1 \%$ QD for 29 days. Safety and tolerability assessments included adverse events (AEs) and local tolerability scales. PK parameters were calculated using non-compartmental analysis. Efficacy assessments included change in PGA, Psoriasis Area and Severity Index score, and \%BSA affected.

Results Twenty-one patients were enrolled. Common AEs were folliculitis, headache, back pain, and pruritus (none led to discontinuation). Tapinarof plasma exposure was low, with the majority of concentrations being below detectable limits. At day 29, 14 patients (73.7\%) had a $\geq 1$-grade improvement in PGA score and six patients (31.6\%) had a $\geq 2$-grade improvement; four patients $(21.1 \%$ ) achieved treatment success (PGA 0 or 1 and $\geq 2$-grade improvement).

Conclusion Tapinarof cream 1\% QD was well tolerated, with limited systemic exposure and significant efficacy at 4 weeks

Stephen C. Piscitelli

steve.piscitelli@dermavant.com

1 Dermavant Sciences, 3300 Paramount Parkway, STE 150, Morrisville, NC 27560, USA

2 Progressive Clinical Research, San Antonio, TX, USA

3 Paddington Testing Company, Inc, Philadelphia, PA, USA

4 DermResearch, Inc, Austin, TX, USA

5 T. Joseph Raoof MD, Inc, Encino, CA, USA

6 Encinitas Research Center, Encino, CA, USA

\section{Key Points}

There is a need for efficacious topical therapies for psoriasis that can be used without restrictions on application site and the extent or duration of treatment, with convenient once-daily administration.

Tapinarof is a first-in-class, non-steroidal, topical therapeutic aryl hydrocarbon receptor modulating agent that is in development for the treatment of psoriasis and atopic dermatitis.

Tapinarof cream 1\% QD has limited systemic exposure under maximal use conditions in adults with extensive plaque psoriasis ( $\geq 20 \%$ body surface area involvement) and has the potential to provide a highly effective treatment option that can be used without restrictions in site/ extent of application and duration of use in accordance with reported findings in longer clinical trials. 
Psoriasis disrupts daily activities such as work attendance, interpersonal relationships, and recreational activities, thereby significantly impacting quality of life. Psoriasis also places a large economic burden on society from healthcare costs to lost productivity [3, 4].

Up to $80 \%$ of patients have mild to moderate psoriasis that is generally managed with topical treatments. Although numerous topical treatments are available, there is a need for options that combine high efficacy with acceptable safety, without treatment duration or body surface area (BSA) restrictions.

Tapinarof is a first-in-class, non-steroidal, topical therapeutic aryl hydrocarbon receptor (AhR) modulating agent that is in development for the treatment of psoriasis and atopic dermatitis. Tapinarof specifically binds to and activates the AhR, a ligand-dependent transcription factor, leading to the downregulation of pro-inflammatory cytokines, including interleukin-17, and induction of expression of skin barrier proteins related to keratinocyte differentiation, including filaggrin and loricrin, thus promoting skin barrier normalization [5-7]. Tapinarof has been evaluated in 18 clinical trials including more than 2200 patients, demonstrating significant efficacy and a favorable safety and tolerability profile [8-10].

The purpose of this trial was to evaluate systemic exposure of tapinarof under conditions that would maximize the potential for drug absorption with the intended use of the product [11].

\section{Materials and Methods}

\subsection{Study Design}

This was a phase IIa, multicenter, open-label maximal use study of tapinarof cream $1 \%$ once daily (QD) in adult patients with extensive ( $\geq 20 \%$ BSA involvement) plaque psoriasis. Patients were enrolled at five clinical sites in the United States (US). After a 34-day screening period, eligible patients applied tapinarof cream 1\% QD from days 1 through 29 to all originally identified affected areas, and any newly appearing plaques. Patients returned to the clinic on days 2, 15, 29, and 30 for study assessments and received phone calls on days 8 and 22. A final in-person follow-up visit occurred 7-10 days after the day 29 visit. On clinic visit days during the treatment period, patients applied the study drug under the supervision of site personnel after assessments had been completed. Full pharmacokinetics $(\mathrm{PK})$ profiles and Holter monitoring were collected on day 1 and on day 29 (with corresponding 24-h time points collected on day 2 and day 30, respectively). Limited PK sampling was performed on day 15. Pretreatment, time-matched Holter monitoring was conducted on day -1 and the morning of day 1. Patients who withdrew from the study before day 30 completed an early termination visit.
Compliance was assessed by completion of a daily patient diary. Patients were considered to be compliant when they applied $80-120 \%$ of the total number of expected doses during the treatment period. Safety was assessed by adverse events (AEs), physical examination, vital signs, and laboratory assessments. Local tolerability was evaluated using investigator-assessed scales, completed at baseline and day 29. Investigators assessed the presence and degree of irritation based on dryness, erythema, and peeling. Efficacy was assessed using a 5-point Physician Global Assessment (PGA) scale, Psoriasis Area and Severity Index (PASI) score, and \%BSA affected.

The study was conducted in accordance with the International Conference on Harmonization of Technical Requirements for Registration of Pharmaceuticals for Human Use Good Clinical Practice and the Declaration of Helsinki 2008. Approval was obtained from the local ethics committee or institutional review board at each trial center. Written informed consent was obtained from each patient prior to the performance of any study-specific procedures. The primary objectives were to evaluate the safety, tolerability, and PK of tapinarof cream 1\% QD in patients with extensive plaque psoriasis. The secondary objectives were to evaluate the efficacy of tapinarof cream 1\% QD in patients with extensive plaque psoriasis and to exclude clinically relevant effects of tapinarof cream 1\% QD on the Q- to T-wave (QT) interval corrected for heart rate (HR) using Fridericia's formula (QTcF), a measure of cardiac repolarization. The key inclusion and exclusion criteria are summarized in Supplementary Table 1 (see the electronic supplementary material).

\subsection{Study Treatments}

Tapinarof cream $1 \%$ was dispensed to patients during clinic visits and administered at home between clinic visits. Patients were instructed to apply tapinarof cream 1\% QD in the morning to all affected areas, including newly appearing plaques and plaques/areas that improved during the study. Patients applied sufficient quantity to cover each lesion completely with a thin layer. Patients were allowed, but not required, to treat palms, fingernails, soles, toenails, and scalp.

\subsection{Study Assessments}

Safety assessments included the frequency and severity of local and systemic AEs, treatment-emergent AEs (TEAEs), serious AEs (SAEs), AEs of special interest (AESIs), brief physical examinations, laboratory values, vital signs, safety electrocardiograms (ECGs), and local tolerability scales. AEs, TEAEs, SAEs, and AESIs were assessed during visits at screening, day -1 , baseline, days $2,15,29,30$, and follow-up, and during phone calls at days 8 and 22. Brief physical examinations were assessed at screening and follow-up. 
Laboratory values were assessed at screening, baseline, day 29, and follow-up. Vital signs were assessed at screening, baseline, and follow-up. Safety ECGs were assessed at screening and follow-up. Local tolerability scales were assessed at baseline and days 15 and 29, with a score of 0 indicating no irritation and a score of 4 indicating very severe irritation (see the electronic supplementary material, Supplementary Table 2). Serial plasma samples for PK analysis were collected on days 1 and 29 at pre-dose, at 1, 2, 3, 4, 5,8 , and $12 \mathrm{~h}$ after dosing, and on days 2 and 30 at $24 \mathrm{~h}$ after dosing. Additional plasma samples for PK analysis were collected on day 15 at pre-dose and at 2 and $4 \mathrm{~h}$ after dosing. PK profiles for tapinarof and tapinarof sulfate (metabolite) were derived on days 1 and 29, and the following PK parameters were calculated, data permitting: area under the curve from time zero to the last quantifiable time point $\left(\mathrm{AUC}_{0-\text { last }}\right)$, maximum plasma concentration $\left(C_{\max }\right)$, time to maximum plasma concentration $\left(T_{\max }\right)$, and elimination half-life $\left(t_{1 / 2}\right)$.

The secondary endpoints were analysis of the mean change from baseline in QT interval corrected for HR using Fridericia's formula $(\Delta \mathrm{QTcF})$ at each post-treatment time point on the sampling day with the higher $C_{\max }$ (day 1); a concentration-QTcF analysis investigating the relationship between tapinarof plasma concentration and $\triangle \mathrm{QTcF}$; and the following efficacy assessments: mean change from baseline to day 29 in PGA, PASI score, and \%BSA affected. Data for QTcF, $\triangle \mathrm{QTcF}$, and other cardiodynamic assessments were obtained from continuous Holter readings collected on days - 1, 1, 2, 29, and 30. All Holter/ECG data were collected using M12R continuous 12-lead digital recorders and the M12A Enterprise Holter System Client (Global Instrumentation, LLC, Manlius, New York, USA). ECG intervals were measured by the core laboratory in a blinded manner using the Expert Precision QT technique. Efficacy assessments (PGA, PASI, and \%BSA affected) were conducted at screening, baseline, and day 29.

Exploratory endpoints included additional cardiodynamic assessments obtained from continuous Holter readings collected on days $-1,1,2,29$, and 30 , including analysis of change in HR from baseline ( $\Delta \mathrm{HR})$, time between the $\mathrm{P}$ - and $\mathrm{R}$-wave (PR) interval ( $\Delta \mathrm{PR})$, and complex between $\mathrm{Q}$ - and $\mathrm{S}$-wave (QRS) interval $(\Delta \mathrm{QRS})$ at each post-treatment time point on the sampling day with the higher $C_{\max }$ (day 1); analysis of categorical outliers for QTcF, HR, PR, and QRS intervals; and frequency of treatment-emergent changes for $\mathrm{T}$-wave morphology and U-wave presence.

\subsection{Data Analysis}

\subsubsection{Safety and Tolerability Analyses}

Safety and tolerability were evaluated by assessment of clinical laboratory tests, physical examinations, vital signs measurements, ECG readings, and local tolerability scales at various time points during the study and by the documentation of AEs. The following were reported as AESIs: contact dermatitis, folliculitis, and headache. All AEs were listed. By-patient data listings were generated for AEs, TEAEs, SAEs, AESIs, and AEs leading to study discontinuation

\subsubsection{Pharmacokinetics (PK) Analysis}

Concentrations of tapinarof and tapinarof sulfate (metabolite) were determined in plasma samples using a validated bioanalytical method. The lower limit of quantitation of the assay, which corresponds to the lowest concentration that can be reliably measured, was $50 \mathrm{pg} / \mathrm{mL}$ for tapinarof and $10 \mathrm{pg} / \mathrm{mL}$ for tapinarof sulfate. All PK analyses were performed using Phoenix ${ }^{\circledR}$ WinNonlin ${ }^{\circledR}$ Version 8.1 (Certara, Princeton, NJ, USA) as part of a 21 CFR Part 11-compliant database system (Pharsight Knowledgebase Server $\left.{ }^{\mathrm{TM}}\right)$. PK parameter estimates for tapinarof on days 1 and 29 were calculated using noncompartmental analysis of the plasma concentration versus time data utilizing the actual sample collection time. $\mathrm{AUC}_{0-\text { last }}$ values were estimated using the linear up-log down method. A minimum number of three data points were required for determination of the $\mathrm{AUC}_{0-\text { last }}$.

\subsubsection{Cardiodynamic Analyses}

All statistical analyses for cardiodynamic assessments were performed using SAS Version 9.4 or higher (SAS Institute, Inc., Cary, NC). In all calculations, zero was substituted for below the quantification limit (BQL) concentrations. The bytime-point analysis for QTcF was based on a linear mixedeffects model with $\Delta \mathrm{QTcF}$ as the dependent variable; time (i.e., post-baseline time point on the study day: categorical), treatment (tapinarof), and time-by-treatment interaction as fixed effects. The by-time-point analysis for HR, PR, and $\mathrm{QRS}$ intervals was based on the $\Delta \mathrm{HR}, \Delta \mathrm{PR}$, and $\Delta \mathrm{QRS}$, respectively, and the same model was used for $\Delta \mathrm{QTcF}$. For the concentration-QTcF analysis, the relationship between tapinarof plasma concentrations and $\Delta \mathrm{QTcF}$ was investigated using a linear mixed-effects modeling approach, with $\Delta \mathrm{QTcF}$ as the dependent variable, time-matched tapinarof plasma concentration as the explanatory variable, a fixed intercept, and a random intercept and slope per patient, when applicable [12].

\subsubsection{Efficacy Analyses}

Efficacy was assessed by PGA, PASI, and \%BSA affected, which were summarized at baseline and day 29. Change and percent change in PGA from baseline to day 29 were 
summarized descriptively and analyzed with a 1-sample $t$ test. The percentage of patients demonstrating treatment success, defined by a PGA score of clear or almost clear ( 0 or 1 , respectively) and a $\geq 2$-grade improvement, was assessed. The percentages of patients with a $\geq 1$-grade improvement and $\mathrm{a} \geq 2$-grade improvement from baseline in PGA were also summarized.

\section{Results}

\subsection{Study Population}

Thirty-six adults with extensive plaque psoriasis were screened; 21 met inclusion/exclusion criteria and initiated treatment with tapinarof cream 1\%; 19 out of $21(90.5 \%)$ completed the trial, one out of $21(4.8 \%)$ withdrew consent, and one out of 21 (4.8\%) was lost to follow-up (Fig. 1). Patient demographics and baseline disease characteristics are summarized in Table 1.

Nineteen of 20 patients (95.0\%) were compliant with treatment. One patient who was noncompliant administered only two applications of the expected 29 and withdrew consent 14 days after their first dose. All others administered between 27 and 30 applications of the study drug. Compliance could not be determined for the patient lost to follow-up as the study diary was not returned.

\subsection{Safety Results}

TEAEs, defined as those arising on or after the first study drug application, were reported in 12 of 21 (57.1\%) patients. The most frequently reported TEAEs were folliculitis (four patients; 19.0\%), headache (four patients; 19.0\%), back pain (two patients; 9.5\%), and pruritus (two patients; 9.5\%). The TEAEs of folliculitis (four out of four patients) and headache (two out of four patients) were considered to be related to study drug.

There were no clinically relevant changes in laboratory values, vital signs, or ECG parameters. There were no deaths or discontinuations due to AEs. One patient experienced a SAE of pancreatitis that was unrelated to study drug.

Tapinarof was well tolerated at application sites. At baseline (day 1), seven patients applied the study drug to sensitive areas that included face, axilla, neck, anal crux, inframammary, and genitalia. No patients applying the product to sensitive areas reported irritation at baseline, day 15 , or day 29 . Overall, local tolerability was improved, with lower tolerability scores reported at day 29 compared to baseline. At day 29, 17 patients had no irritation and two patients had mild irritation.

\subsection{PK Results}

Mean tapinarof plasma concentrations versus time curves on day 1 and day 29 are shown in Fig. 2. Tapinarof concentrations were highest on day 1 , with $T_{\max }$ between 2 and $5 \mathrm{~h}$ post-dose. On day 29, tapinarof concentrations were approximately tenfold lower than those observed on day 1 , with slight fluctuations in concentrations at the measured time points. Across all patients, tapinarof plasma exposure was low, with most samples (284 out of 418 [67.9\%]) having concentrations BQL $(<50 \mathrm{pg} / \mathrm{mL})$. Tapinarof sulfate concentrations in all PK samples were BQL $(<10 \mathrm{pg} / \mathrm{mL})$.

The PK parameters for tapinarof were calculated using available tapinarof concentration data (Table 2). On day 1 , $C_{\max }$ values ranged from 0 to $4680 \mathrm{pg} / \mathrm{mL}$ (mean of $898 \mathrm{pg}$ / $\mathrm{mL}$, median of $181 \mathrm{pg} / \mathrm{mL}$ ). $\mathrm{AUC}_{0-\text { last }}$ values on day 1 ranged from 26.8 to $22,113 \mathrm{pg} \cdot \mathrm{h} / \mathrm{mL}$ (geometric mean of $861 \mathrm{pg} \cdot \mathrm{h} / \mathrm{mL}$ ). Tapinarof exposure decreased over the course of the trial, with day 29 mean and median $C_{\max }$ values of $116 \mathrm{pg} / \mathrm{mL}$ and $59.4 \mathrm{pg} / \mathrm{mL}$, respectively, and a geometric mean $\mathrm{AUC}_{0-\text { last }}$ value of $321 \mathrm{pg} \cdot \mathrm{h} / \mathrm{mL}$. Geometric mean $C_{\max }$ was not calculated on days 1 or 29 due to the high percentage of zero values in the data set. $t_{1 / 2}$ could only be calculated in two patients $(9.5 \%)$ on day 1 and no patients on day 29 .

\subsection{Cardiodynamic Results}

\subsubsection{Effect on Cardiac Repolarization}

In the by-time-point analysis, $\Delta \mathrm{QTcF}$ was negative at all post-dose time points on day 1 (the sampling day with the higher $C_{\max }$ ), ranging from $-7.2 \mathrm{~ms}$ to $-1.1 \mathrm{~ms}$. There were three patients (seven time points) with QTcF between 450

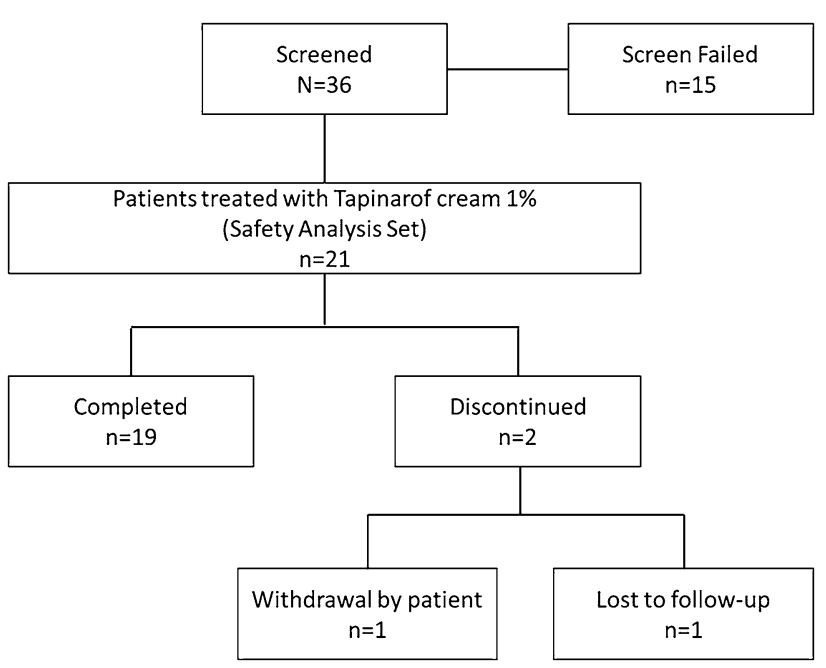

Fig. 1 Patient disposition 
Table 1 Patient demographics and baseline disease characteristics

\begin{tabular}{ll}
\hline Characteristic & Tapinarof cream 1\% QD \\
& $N=21$ \\
\hline Age, years, mean (SD) ${ }^{\mathrm{a}}$ & $51.8(13.91)$ \\
Male, $n(\%)$ & $13(61.9 \%)$ \\
Female, $n(\%)$ & $8(38.1 \%)$ \\
Childbearing potential =yes & $2(25.0 \%)$ \\
Ethnicity, $n(\%)$ & \\
Hispanic or Latino & $9(42.9 \%)$ \\
Not Hispanic or Latino & $12(57.1 \%)$ \\
Race,$n(\%)$ & \\
American Indian or Alaska Native & $1(4.8 \%)$ \\
Black or African American & $3(14.3 \%)$ \\
White & $16(76.2 \%)$ \\
Not reported & $1(4.8 \%)$ \\
Height (cm), mean (SD) & $171.62(9.758)$ \\
Weight (kg), mean (SD) & $97.51(24.619)$ \\
BMI (kg/m $\left.{ }^{2}\right)$, mean (SD) & $33.065(7.8922)$ \\
PGA, $n(\%)$ & \\
3-moderate & $13(61.9 \%)$ \\
4-severe & $8(38.1 \%)$ \\
Total \%BSA affected & $27.20(7.481)$ \\
Mean (SD) & $25.5(20.5,46.0)$ \\
Median (minimum, maximum) & \\
Total PASI & $24.65(7.146)$ \\
Mean (SD) & \\
Median (minimum, maximum) & \\
\hline
\end{tabular}

$\% B S A \%$ body surface area, BMI body mass index, PASI Psoriasis Area Severity Index, $P G A$ Physician Global Assessment, $Q D$ once daily, $S D$ standard deviation

${ }^{a}$ Age was calculated in years relative to the informed consent date

${ }^{\mathrm{b}} \mathrm{A}$ patient could report more than one race

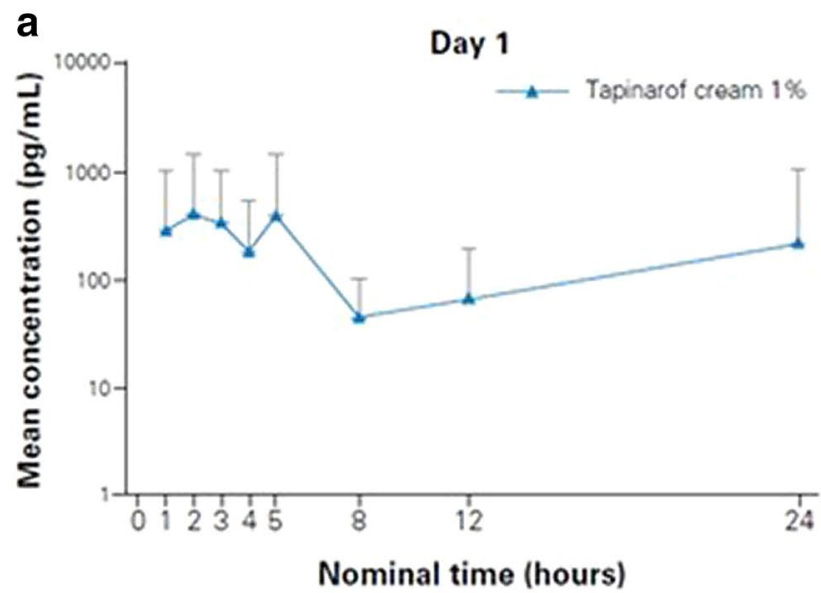

and $480 \mathrm{~ms}$ and no patients with QTcF $>480 \mathrm{~ms}$. There was one patient (one time point) with $\Delta \mathrm{QTcF}$ between 30 and $60 \mathrm{~ms}$ and no patients with $\triangle \mathrm{QTcF}>60 \mathrm{~ms}$.

In the concentration-QTcF analysis, a linear model with an intercept was fitted for tapinarof plasma concentrations. The estimated slope of tapinarof plasma concentration in the concentration-QTcF relationship was not statistically significant, shallow, and slightly negative: $-0.00016 \mathrm{~ms}$ per pg/ $\mathrm{mL}$ (90\% confidence interval $[\mathrm{CI}]-0.002172$ to 0.001844$)$, with an intercept of $-3.3 \mathrm{~ms}(90 \% \mathrm{CI}-6.03$ to -0.62$)$. The effect on $\triangle \mathrm{QTcF}$ can be predicted to be $-3.35 \mathrm{~ms}(90 \% \mathrm{CI}$ -6.02 to -0.67$)$. Based on this concentration-QTcF analysis, a $\Delta$ QTcF exceeding $10 \mathrm{~ms}$ can be excluded within the observed range of tapinarof plasma concentrations up to $\sim 4600 \mathrm{pg} / \mathrm{mL}$.

No clinically relevant effects on any other ECG parameters were observed.

\subsection{Efficacy Results}

Nineteen of $21(90.5 \%)$ patients had assessments at baseline and day 29 and were included in the evaluation of efficacy. A summary of efficacy results is shown in Table 3.

\subsubsection{Physician Global Assessment (PGA)}

Of the 21 enrolled patients, 13 patients (61.9\%) had a PGA score of 3 (moderate) and eight patients $(38.1 \%)$ had a PGA score of 4 (severe) at baseline. Of the 19 patients that completed the study, 12 patients $(63.2 \%)$ had a PGA score of 3 (moderate) and seven patients $(36.8 \%$ ) had a PGA score of 4 (severe) at baseline. At day 29, five patients (26.3\%) had moderate and one patient (5.3\%) had severe disease. Most patients had improved to a PGA score of 2

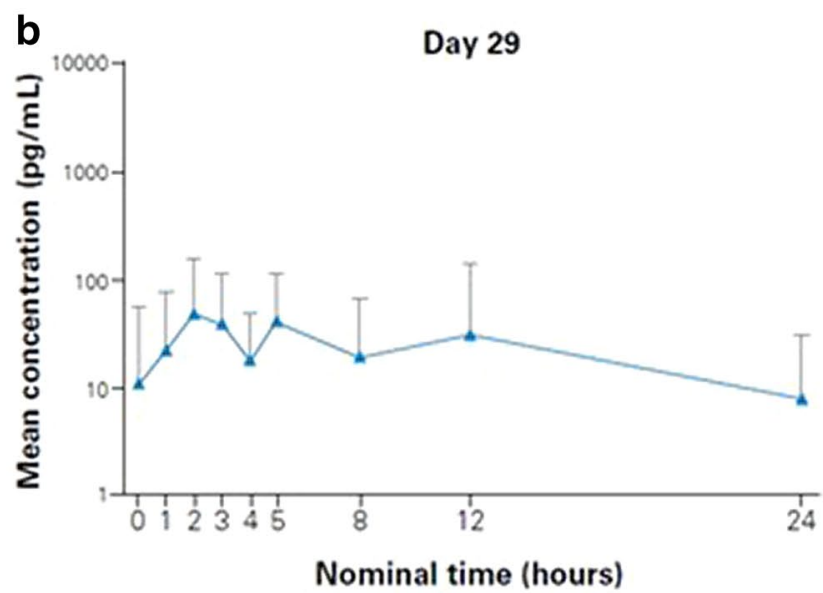

Fig. 2 Mean (+/- standard deviation) tapinarof plasma concentration vs time curves on a day 1 (baseline) and b day 29 . All tapinarof sulfate concentrations were below the LLOQ of $10 \mathrm{pg} / \mathrm{mL}$. Tapinarof LLOQ was $50 \mathrm{pg} / \mathrm{mL}$. LLOQ lower limit of quantitation 
Table 2 Pharmacokinetics parameter estimates for tapinarof by day

\begin{tabular}{|c|c|c|c|c|c|c|c|c|c|}
\hline PK parameter ${ }^{\mathrm{a}}$ & $N$ & Mean & SD & $\mathrm{CV} \%$ & Min & Median & $\operatorname{Max}$ & Geo. mean & Geo. CV\% \\
\hline \multicolumn{10}{|l|}{ Baseline (day 1) } \\
\hline$C_{\max }(\mathrm{pg} / \mathrm{mL})$ & 21 & 898 & 1448 & 161.2 & 0.0 & 181 & 4680 & $\mathrm{NC}^{\mathrm{b}}$ & $\mathrm{NC}^{\mathrm{b}}$ \\
\hline$T_{\max }(\mathrm{h})$ & 18 & 4.39 & 5.48 & 124.8 & 1.1 & 3.10 & 23.8 & 2.99 & 94.55 \\
\hline$T_{\text {last }}(\mathrm{h})$ & 18 & 9.50 & 7.85 & 82.6 & 1.2 & 8.04 & 24.2 & 6.48 & 123.4 \\
\hline $\mathrm{AUC}_{0-\text { last }}(\mathrm{pg} \cdot \mathrm{h} / \mathrm{mL})$ & 17 & 4067 & 6331 & 155.7 & 26.8 & 2095 & 22,113 & 861 & 1067 \\
\hline$t_{1 / 2}(\mathrm{~h})$ & 2 & 6.41 & 0.691 & 10.78 & 5.9 & 6.41 & 6.9 & 6.39 & 10.83 \\
\hline \multicolumn{10}{|l|}{ Day $29^{c}$} \\
\hline$C_{\max }(\mathrm{pg} / \mathrm{mL})$ & 19 & 116 & 148.4 & 127.85 & 0.0 & 59.4 & 467 & - & - \\
\hline$T_{\max }(\mathrm{h})$ & 11 & 4.02 & 3.005 & 74.71 & 1.1 & 3.17 & 12.2 & 3.31 & 70.30 \\
\hline$T_{\text {last }}(\mathrm{h})$ & 11 & 7.39 & 6.650 & 90.03 & 2.2 & 5.13 & 24.1 & 5.54 & 88.32 \\
\hline $\mathrm{AUC}_{0 \text {-last }}(\mathrm{pg} \cdot \mathrm{h} / \mathrm{mL})$ & 11 & 609 & 645.9 & 106.1 & 29.7 & 279 & 1994 & 321 & 220.0 \\
\hline$t_{1 / 2}(\mathrm{~h})$ & 0 & - & - & - & - & - & - & - & - \\
\hline
\end{tabular}

BQL values (concentrations $<50 \mathrm{pg} / \mathrm{mL}$ ) before the first quantifiable concentration were set to zero. BQL values after the first quantifiable concentration and followed by a value above the lower limit of quantification were set as missing. If two consecutive BQL values occurred after $T_{\max }$, the subsequent values were imputed as missing

$A U C_{0-\text { last }}$ area under the curve from time zero to the last quantifiable time point, $B Q L$ below the quantification limit, $C_{\max }$ maximum plasma concentration, $C V \%$ coefficient of variation percentage, Geo. geometric, Max maximum, Min minimum, $N C$ not calculated, $P K$ pharmacokinetics, $S D$ standard deviation, $t_{1 / 2}$ elimination half-life, $T_{\text {last }}$ time of the last measurable concentration, $T_{\max }$ time to maximum plasma concentration

${ }^{a}$ Missing sampling or concentration values were not imputed, but left missing in the calculation of derived PK parameters. Patients with a minimum of three post-dose time points were required for determination of $\mathrm{AUC}_{0 \text {-last }}$. A minimum of three descending time points with quantifiable plasma concentrations after $C_{\max }$ were used in the estimation of the terminal phase rate constant $\left(\lambda_{z}\right)$, for the determination of the $t_{1 / 2}$. $t_{1 / 2}$ was reported wherever the adjusted $R^{2}$ value was $>0.7$

${ }^{\mathrm{b}}$ Geometric mean and geometric CV\% were not calculated for $C_{\max }$ on days 1 or 29 due to the greater than expected number of zero values in the data set

${ }^{\mathrm{c}}$ One patient had their 24-h sample on day 30 (visit 7) collected outside the predetermined sample collection window; therefore, the sample was excluded from the analysis

(mild disease; nine patients; 47.4\%) or 1 (almost clear; four patients; $21.1 \%$ ). The mean (standard deviation [SD]) change from baseline in PGA score at day 29 was -1.2 (1.0) $(P<0.0001)$.

Fourteen patients $(73.7 \%)$ had a $\geq 1$-grade improvement in PGA score at day 29 and six patients (31.6\%) experienced a $\geq 2$-grade improvement from baseline, demonstrating a shift of patients from more severe disease to milder disease at day 29. Despite only a 4-week treatment period, four patients $(21.1 \%)$ met the criteria for treatment success, which required a PGA score of 0 (clear) or 1 (almost clear) and a $\geq 2$-grade improvement from baseline.

\subsubsection{Psoriasis Area and Severity Index (PASI)}

At baseline, the mean (SD) total PASI score was 24.65 (7.146). At day 29, a significant mean change in PASI score from baseline of -15.14 (95\% CI -19.61 to -10.66$)$ and a mean percent change from baseline of $-59.56 \%$ (95\% CI -73.53 to -45.59 ) were observed. Eighteen of 19 patients (94.7\%) demonstrated improvement in PASI score during the trial.

\subsection{3 \%BSA Affected}

At baseline, the mean \%BSA (SD) affected was 27.20 (7.481). A significant mean change in \% BSA affected from baseline of -14.44 (95\% CI -19.84 to -9.04$)$ and mean percent change from baseline of $-49.77 \%$ (95\% CI -65.45 to -34.09 ) were observed at day 29. Seventeen of 19 patients $(89.5 \%)$ demonstrated improvement in \%BSA affected during the trial.

\section{Discussion}

This trial assessed the PK, safety, cardiodynamics, and efficacy of tapinarof under conditions of maximal use in patients with plaque psoriasis with $\geq 20 \%$ BSA of disease. This coverage area represents the extreme of drug application in this disease and serves as a maximum exposure scenario to evaluate drug absorption following topical application.

Safety and tolerability are key determining factors in whether patients adhere to their prescribed regimen of topical medications. The favorable safety profile of tapinarof 
Table 3 Efficacy following 4 weeks of treatment

\begin{tabular}{ll}
\hline Efficacy parameter & $\begin{array}{c}\text { Tapinarof cream 1\% QD } \\
(N=19)\end{array}$ \\
\hline Patients with $\geq 1$-grade improvement in PGA score, $n(\%)$ & $14(73.7 \%)$ \\
Patients with $\geq 2$-grade improvement in PGA score, $n(\%)$ & $6(31.6 \%)$ \\
Patients with a PGA score of 0 or 1 and a $\geq 2$-grade improvement in PGA score, $n(\%)$ & $4(21.1 \%)$ \\
Mean change in PGA from baseline to day 29 (SD) & $-1.2(1.0)$ \\
Mean \% change in PASI score from baseline to day 29 (SD) & $-59.56(28.981)$ \\
Mean \% change in BSA affected from baseline to day 29 (SD) & $-49.77(32.529)$ \\
Patients who achieved PASI75, $n(\%)$ & $7(36.8 \%)$ \\
\hline
\end{tabular}

Nineteen of 21 (90.5\%) patients had assessments at baseline and day 29 and were included in the evaluation of efficacy

$B S A$ body surface area, PASI Psoriasis Area Severity Index, PASI75 $\geq 75 \%$ improvement in PASI score from baseline, PGA Physician Global Assessment, $Q D$ once daily, $S D$ standard deviation

is consistent with the low systemic absorption following topical dosing. There were no treatment interruptions or discontinuations due to TEAEs. Folliculitis and headache were the most common TEAEs, reported in four patients each. Severity of folliculitis was mild (grade 1) in three patients and moderate (grade 2) in one patient, with no patients discontinuing application of tapinarof due to folliculitis. Folliculitis in this study was consistent with patterns observed in the pivotal studies, in which most events were mild and associated with low discontinuation rates regardless of duration of treatment [10]. Based on the role of tapinarof in regulating skin barrier protein expression, we hypothesize that the reported events of folliculitis may be primarily associated with follicular cornification and plugging resulting from the upregulation of stratum corneum components related to keratinocyte differentiation. Although the exact mechanism of headache is unclear, we believe that this AE is not due to systemic absorption of tapinarof, based on the minimal systemic exposure demonstrated in the current study.

Tapinarof tolerability scores indicated no or mild irritation in most cases, including no irritation observed when applied in sensitive areas. This safety profile represents a significant advantage over other topical products, including retinoids, vitamin $\mathrm{D}$ analogs, and corticosteroids, which have restrictions on chronic use, application sites, or both.

Across all patients, tapinarof plasma exposure was low and the majority of samples were BQL. Tapinarof exposure decreased over the course of the study, with mean $C_{\max }$ values of $898 \mathrm{pg} / \mathrm{mL}$ and $116 \mathrm{pg} / \mathrm{mL}$ on days 1 and 29, respectively. The reason for decreased tapinarof systemic exposure over time is unknown, but may be due to skin barrier function repair. No relationship between tapinarof exposure and $\%$ BSA of disease was observed. For example, the patient with the highest \%BSA affected (46\%) had mostly undetectable concentrations, whereas another patient at the low end of the \%BSA affected range (22\%) had an almost complete
PK profile. There were no patients with measurable tapinarof sulfate (metabolite) concentrations even with a sensitive assay, confirming that tapinarof is the major circulating component in plasma.

Safety evaluations included assessments of QT interval prolongations and other cardiodynamic parameters, as required by the Food and Drug Administration for all investigational drugs. No evidence of QT interval prolongation or effects on other ECG parameters was observed by matched time point analysis or concentration-QTcF analysis. This evaluation in patients with psoriasis is useful to confirm the lack of a potential QT interval effect with tapinarof, given that it may be challenging to achieve plasma concentrations in healthy volunteers with intact skin in a thorough QT study. These data are consistent with those of nonclinical studies that were conducted in minipigs, with up to $6 \%$ tapinarof cream showing no effect on QT interval or HR. Furthermore, the in vitro $50 \%$ inhibitory concentration for $h E R G$ (human ether-a-go-go-related gene) inhibition is $>20,000$-fold the free plasma concentration at the clinical $C_{\max }$.

Tapinarof has demonstrated efficacy and safety in two phase III clinical trials for the treatment of plaque psoriasis in adults [10]. In these pivotal trials, the treatment duration was 12 weeks. Patients in the current study were treated for only 29 days because the primary objective of maximal use trials is assessment of safety and PK. Despite the shortened treatment course, improvement in PGA and PASI scores were observed. Six patients $(32 \%)$ had $\geq 2$-grade improvement in the PGA score, and four patients (21\%) had a PGA score of clear or almost clear (0 or 1, respectively) and a $\geq 2$-grade improvement. Similarly, seven patients (37\%) achieved a $75 \%$ improvement in the PASI score over 4 weeks, and the percent decrease in BSA affected was approximately $50 \%$ from baseline to day 29 . These data demonstrate the activity of tapinarof in plaque psoriasis and the rapid onset of effect. 
The results from this study demonstrate that tapinarof cream $1 \%$ QD is generally well tolerated, has limited systemic exposure under maximal use conditions in adults with plaque psoriasis with $\geq 20 \%$ BSA involvement, does not have an effect on QT interval or other ECG parameters, and demonstrates activity on psoriatic plaques after only 29 days of treatment.

This phase IIa trial was primarily designed to assess the safety and PK of tapinarof cream under maximal use conditions. Consequently, the relatively small sample size $(n=21)$ of patients with extensive plaque psoriasis, and the focus on safety and PK endpoints, means that conclusions on efficacy are limited, and may not be generalized to the full spectrum of patients, including those with less severe disease, which are included in larger longer trials $[10,13]$.

Tapinarof may address many of the limitations of the current topical therapeutic options for psoriasis. It has the potential to be used across the disease spectrum, providing physicians and patients with a highly effective treatment option that can be used chronically without restrictions in duration of treatment, application site, or BSA treated, with a durable effect after treatment discontinuation [8], a novel mechanism of action [5-7], a favorable tolerability profile [10], and a PK profile associated with plasma concentrations below the threshold for drug-drug interaction studies [14].

Supplementary Information The online version contains supplementary material available at https://doi.org/10.1007/s40257-021-00641-4.

Acknowledgements The authors thank the participating investigators, patients, and their families, as well as colleagues involved in the conduct of the study. Editorial and medical writing support under the guidance of the authors was provided by ApotheCom, UK, and was funded by Dermavant Sciences, Inc. in accordance with Good Publication Practice (GPP3) guidelines (Ann Intern Med. 2015;163:461-4).

\section{Declarations}

Funding Research was funded by Dermavant Sciences, Inc.

Conflict of interest JEJ, WDM, MM, SCP, MCS, GT, and TW are employees of Dermavant Science, Inc. LCP is an investigator for Dermavant Science, Inc. JDB is an investigator for Allergan, Aclaris Therapeutics, Galderma USA, Brickell Biotech, Derma Therapeutics, Endo International, LEO Pharmaceuticals, Cara Therapeutics, Arcutis, Boston Pharmaceuticals, Atacama Therapeutics, Biofrontera, BristolMyers Squibb, RAPT Therapeutics, NFlection Therapeutics, Incyte Corporation, and Bellus Health. MSL is an investigator for Abbvie, Asana, Pfizer, Eli Lilly, AstraZeneca, Leo, Arena, Dermira, Cara, Bellus, Foamix, Arcutis, Incyte, UCB, and Boehringer Ingelheim. TJR has no conflicts of interest to disclose.

Availability of data and material The data that support the findings of this study are proprietary and are not publicly available. The data may be made available upon reasonable request and with permission from Dermavant after marketing approval is obtained, but restrictions will apply to the availability of these data.
Code availability Not applicable.

Authors' contributions Conceptualization: GT, JEJ, LCP, MM, and SCP. Data curation: GT, JEJ, and WDM. Formal analysis: JEJ, WDM, MM, MCS, and TW. Funding acquisition: JEJ and SCP. Investigation: JDB, JEJ, LCP, MSL, SCP, and TJR. Methodology: GT, JEJ, MM, WDM, SCP, and TW. Project administration: GT, JEJ, MM, and WDM. Resources: SCP. Software: WDM. Supervision: GT, JEJ, MM, WDM, and SCP. Validation: WDM. Visualization: JEJ, MM, and SCP. Writing-original draft preparation: GT, JEJ, MM, WDM, SCP, and TW. Writing - review and editing: JEJ, WDM, MM, SCP, MCS, GT, TW, LCP, JDB, MSL, and TJR.

Ethics approval This study was approved by the central Institutional Review Board, Advarra, and conducted in accordance with International Conference on Harmonization of Technical Requirements for Registration of Pharmaceuticals for Human Use Good Clinical Practice and all applicable subject privacy requirements, and the ethical principles that are outlined in the Declaration of Helsinki 2008.

Consent to participate Written informed consent was obtained from all individual participants in the study.

Open Access This article is licensed under a Creative Commons Attribution-NonCommercial 4.0 International License, which permits any non-commercial use, sharing, adaptation, distribution and reproduction in any medium or format, as long as you give appropriate credit to the original author(s) and the source, provide a link to the Creative Commons licence, and indicate if changes were made. The images or other third party material in this article are included in the article's Creative Commons licence, unless indicated otherwise in a credit line to the material. If material is not included in the article's Creative Commons licence and your intended use is not permitted by statutory regulation or exceeds the permitted use, you will need to obtain permission directly from the copyright holder. To view a copy of this licence, visit http://creativecommons.org/licenses/by-nc/4.0/.

\section{References}

1. Parisi R, Symmons DPM, Griffiths CEM, Ashcroft DM. Global epidemiology of psoriasis: a systematic review of incidence and prevalence. J Investig Dermatol. 2012;133:377-85.

2. Boehncke WH, Schon MP. Psoriasis. Lancet. 2015;386:983-94.

3. Krueger G, Koo J, Lebwohl M, Menter A, Stern RS, Rolstad T. The impact of psoriasis on quality of life: results of a 1998 National Psoriasis Foundation patient membership survey. Arch Dermatol. 2001;137:280-4.

4. Bickers DR, Lim HW, Margolis D, Weinstock MA, Goodman C, Faulkner E, et al; American Academy of Dermatology Association; Society for Investigative Dermatology. The burden of skin diseases: 2004 a joint project of the American Academy of Dermatology Association and the Society for Investigative Dermatology. J Am Acad Dermatol. 2006;55:490-500.

5. Smith SH, Jayawickreme C, Rickard DJ, Nicoeme E, Gui T, Simmons $\mathrm{C}$, et al. Tapinarof is a natural AhR agonist that resolved skin inflammation in mice and humans. J Investig Dermatol. 2017;137:2110-9.

6. Furue M, Hashimoto-Hachiya A, Tsuji G. Aryl hydrocarbon receptor in atopic dermatitis and psoriasis. Int J Mol Sci. 2019;20:5424.

7. Bissonnette R, Stein Gold L, Rubenstein DS, Tallman AM, Armstrong A. Tapinarof in the treatment of psoriasis: a review of the unique mechanism of action of a novel 
therapeutic AhR modulating agent (TAMA). J Am Acad Dermatol. 2021;84:1059-67.

8. Robbins K, Bissonette R, Maeda-Chubachi T, Ye L, Peppers J, Gallagher K, et al. Phase 2, randomized dose-finding study of tapinarof (GSK2894512 cream) for the treatment of plaque psoriasis. J Am Acad Dermatol. 2019;80:714-21.

9. Peppers J, Paller AS, Maeda-Chubachi T, Wu S, Robbins K, Gallagher J, et al. A phase 2 randomized dose-finding study of tapinarof (GSK2894512 cream) for the treatment of atopic dermatitis. J Am Acad Dermatol. 2019;80:89-98.

10. Lebwohl M, Stein Gold L, Strober B, Armstrong A, Hong HC-H, Kircik L, et al. Tapinarof cream 1\% QD for the treatment of plaque psoriasis: efficacy and safety in two pivotal phase 3 trials. SKIN J Cutan Med. 2020;4(6):s75.

11. Bashaw ED, Tran DC, Shukla CG, Liu X. Maximal usage trial: an overview of the design of systemic bioavailability trial for topical dermatological products. Ther Innov Regul Sci. 2015;49:108-15.
12. Garnett C, Bonate PL, Dang Q, Ferber G, Huang D, Liu J, et al. Scientific white paper on concentration-QTc modeling. J Pharmacokinet Pharmacodyn. 2018;45:383-97.

13. Strober B. Tapinarof cream $1 \%$ once daily for plaque psoriasis: interim analysis of a long-term extension trial of a novel therapeutic aryl hydrocarbon receptor modulating agent. Poster presentation presented at: Innovations in Dermatology Virtual Spring Conference; March 16-20, 2021.

14. US Food and Drug Administration (FDA). In vitro drug interaction studies - cytochrome P450 enzyme- and transporter-mediated drug interactions guidance for industry. US FDA Center for Drug Evaluation and Research (CDER). January 2020 Clinical Pharmacology. https://www.fda.gov/regulatory-information/search-fdaguidance-documents. Accessed 21 April 2021. 